\title{
TOXOPLASMOSIS COMO AGENTE CAUSAL DE ABORTOS EN ALPACAS
}

\author{
Toxoplasmosis as a Cause of Abortion in Alpacas
}

\author{
Nicasio Valencia M. ${ }^{1}$, Amanda Chávez V., ${ }^{2,3}$, Mario García P. ${ }^{4}$, Francisco Suárez A. ${ }^{5}$ y \\ E. Casas A. ${ }^{2}$
}

\section{Resumen}

El estudio tuvo por objetivo determinar la asociación del T. gondii con los abortos en el primer, segundo y tercer trimestre de gestación, así como su relación con la mortalidad perinatal en la alpaca. El estudio se realizó en el Centro de Investigación y Desarrollo de Camélidos Sudamericanos-Lachocc de la Universidad Nacional de Huancavelica, a una altura de 4450 msnm, durante la campaña reproductiva de enero de 1999 a marzo de 2000, a través del monitoreo de anticuerpos de $T$. gondii, mediante la técnica de Hemoaglutinación Indirecta (HAI). Se evaluaron 332 alpacas hembras previo al empadre obteniéndose una seroprevalencia de 36.5\% (121/332). Con 108 alpacas seronegativas se siguió su seroconversión obteniéndose una incidencia acumulada de 25\% (27/108) a los 270 días después del empadre. La tasa de fertilidad total fue 70.4\% (76/108), estando infectadas por $T$. gondii el $27.6 \%$ (21/76) sin haber diferencia significativa en fertilidad entre animales positivos y negativos a $T$. gondii. Las tasas de aborto para los periodos de 90 a 180, 181 a 270, y 271 a 330 días de gestación fueron de 6.6, 5.6 y 7.6\%, respectivamente, y la tasa de mortalidad perinatal fue $8.1 \%$ sin haber diferencia estadística a la prueba de Fisher entre alpacas negativas e infectadas por $T$. gondii. Además, se encontró que la primoinfección de $T$. gondii en alpacas hembras es independiente de la edad pero que las alpacas seropositivas presentan 3.3 veces mayor riesgo de aborto que las seronegativas.

Palabras clave: Toxoplasma gondii, camélidos, fetos, llama, Huancavelica

\section{AbSTRact}

The objective of the present study was to determine the relationship between $T$. gondii with the occurrence of abortions en the first, second and third trimester of gestation, as well as the association with perinatal mortality. The study was conducted at the Research and Development Centre of South American Camelides, Universidad Nacional de Huancavelica, located at 4450 masl in Lachocc, from January 1999 till March 2000, through the monitoring of antibodies against Toxoplasma gondii using the Indirect

${ }^{1}$ Laboratorio de Sanidad Animal, EAP de Zootecnia, Universidad Nacional de Huancavelica. E-mail: valencian95@hotmail.com

${ }^{2}$ Laboratorio de Microbiología y Parasitología Veterinaria, ${ }^{5}$ Laboratorio de Medicina Veterinaria Preventiva, Facultad de Medicina Veterinaria, Universidad Nacional Mayor de San Marcos, Lima

${ }^{3}$ E-mail: achavezvg@gmail.com

${ }^{4}$ Facultad de Veterinaria y Zootecnia, Universidad Peruana Cayetano Heredia, Lima 
Haemagglutination Test (IHA). The initial seroprevalence of $T$. gondii was 36.5\% (121/ 332). The follow-up of 108 seronegative animals to evaluate serconversion resulted in an accumulate incidence of $25 \%(27 / 108)$ at 270 days after breeding. Fertility rate was $70.4 \%$ (76/108), and among them, $27.6 \%$ (21/76) were infected by $T$. gondii whereas $72.4 \%$ did not seroconverted; however, there was not statistical difference in fertility between positive and negative animals to T. gondii. Abortion rates for the periods 90-80, 181-270, and 271-330 days of gestation were 6.6, 5.6, and 7.6\% respectively, and the perinatal mortality was $8.1 \%$ and without statistical difference using the Fisher Test between positive and negative animals to $T$. gondii. Additionally, prime infection by $T$. gondii in female alpacas showed to be independent of age, but the risk factor indicated that the probability to get an abortion among seropositive female alpacas was 3.3 higher than in seronegative ones.

Key words: Toxoplasma gondii, camelids, fetuses, lama, Huancavelica

\section{INTRODUCCIÓN}

Los problemas reproductivos y de salud se consideran como los principales limitantes para la crianza de alpacas (Fernández Baca, 1971), ya que ocasionan baja tasa de natalidad (50\%) y una alta tasa de mortalidad, que varía entre 7.4 y $51.2 \%$ (Ameghino et al., 1991; Novoa, 1991), 1legando en algunos casos hasta el $80 \%$ (Melo, 1997), sobre todo en animales jóvenes. A ello se suma que una hembra sólo produce cuatro crías en promedio durante su vida productiva (Velásquez y Novoa, 1999).

La mortalidad de crías constituye una seria limitación técnica y económica, tanto para los trabajos de selección por la pérdida de valioso potencial genético, como para los reemplazos pues ocasiona un retraso en el crecimiento de la población alpaquera (Ameghino y DeMartini, 1991).

Los agentes abortivos en alpacas y ovinos pueden ocasionar muerte embrionaria, abortos, malformaciones congénitas, parto de nonatos o de crías débiles que mueren a las pocas horas de nacidas (Leguía, 1990; Rosadio y Ameghino, 1999). Las alpacas raramente muestran signos clínicos previos al aborto, no hay retención de placenta y aparentemente se encuentran saludables, y solo se reconoce el aborto al hallar el feto en el corral o en el campo de pastoreo.
En el marco de la salud animal, una de las infecciones parasitarias zoonóticas de mayor difusión en la naturaleza, es la toxoplasmosis. Esta enfermedad, ocasionada por el Toxoplasma gondii, constituye una de las principales causas de infertilidad, expresada en mortalidad embrionaria temprana, abortos, mortalidad perinatal y mortalidad neonatal en ovinos, caprinos, porcinos, animales silvestres (Tenter et al., 2000) y, probablemente, en camélidos sudamericanos (Leguía et al., 1988). En base a esto, el presente estudio tuvo por objetivo determinar la asociación del $T$. gondii con los abortos en el primer, segundo y tercer trimestre de gestación, así como su relación con la mortalidad perinatal.

\section{MATERIALES Y Métodos}

\section{Localización del Estudio}

El estudio se llevó a cabo entre enero de 1999 hasta marzo de 2000 en el Centro de Investigación y Desarrollo de Camélidos Sudamericanos (CIDCS) - Lachocc, de la Universidad Nacional de Huancavelica (UNH), ubicado en el paraje de Tucumachay, distrito, provincia y región de Huancavelica, a una altitud de $4450 \mathrm{msnm}$. En la zona, las mayores temperaturas se registran durante los meses de mayor pluviosidad (diciembre abril), y los meses más fríos corresponden de 
junio a agosto. La temperatura promedio anual varía entre 4 y $21{ }^{\circ} \mathrm{C}$ y la precipitación media anual es de 990 mm (SENAMI, 2005).

\section{Procedimiento Experimental}

Se hizo una primera selección de 332 alpacas hembras de las razas Huacaya y Suri reproductivamente hábiles, y entre ellas se eligieron a 150 animales de 2 a 7 años de edad, que se encontraban libres de anticuerpos antiT. gondii. Este número se redujo a 108 debido a una saca de emergencia realizada por la UNH a los tres meses de iniciado el estudio.

Con 108 alpacas hembras seronegativas a $T$. gondii se hizo un estudio de cohorte única, considerándose como día " 0 " la fecha del inicio del empadre. Se tomaron muestras de sangre por punción directa de la vena yugular los días $0,4,8,12,16,90,180$ y 270 ; el suero resultante, luego de centrifugar las muestras a $3000 \mathrm{rpm}$ por 5 minutos, fue conservado en congelación a $-20{ }^{\circ} \mathrm{C}$ hasta su análisis por la prueba de hemoaglutinación indirecta (HAI), utilizándose un kit comercial (Toxotest Wiener Laboratorios, 2000, Rosario, Argentina). Se consideró como positivo a los valores HAI $\geq 1 / 16$ (punto de corte).

Se hizo un empadre de tipo "alterno o rotatorio" con $6 \%$ de machos por un periodo de 60 días, y se hicieron exámenes de ecografía y balotaje del abdomen hasta los 330 días para determinar la gestación. Los resultados se evaluaron a través de indicadores epidemiológicos como prevalencia, incidencia, tasa de fertilidad, tasa de aborto y tasa de mortalidad perinatal.

\section{Análisis de Datos}

Se utilizó la prueba de Chi Cuadrado para determinar la asociación de infección de $T$. gondi con estado reproductivo, así como la primo infección con edad de las alpacas. Además, se usó la Prueba Exacta de Fisher para determinar la asociación entre infección de $T$. gondii con abortos y mortalidad perinatal (Daniel, 2004), y la Ecuación de Estimación Generalizada (G.E.E.) para el análisis en estudios longitudinales con alpacas preñadas seropositivas y seronegativas en relación a abortos (Zeger y Liang, 1986).

\section{Resultados}

La seroprevalencia de $T$. gondii en alpacas hembras del CIDCS-Lachocc, antes del inicio de la campaña de empadre del año 1999-2000 fue 36.5\% (121/332). Luego, la incidencia de $T$. gondii a los 0-16, 17$90,91-180$ y $181-270$ días post empadre fue de $5.6 \%(6 / 108), 7.5 \%(8 / 102), 8.5 \%$ (8/94), y $5.8 \%(5 / 86)$, respectivamente, siendo la incidencia acumulada a los 270 días de $25.0 \%$ (27/108).

El porcentaje de gestación fue de $70.4 \%$ (Cuadro 1), y de estas, el 27.6\% (21/76) se infectaron con $T$. gondii en algún momento de su preñez; sin embargo, la seroconversión no afectó significativamente la fertilidad de los animales, según la prueba de Chi Cuadrado.

Las tasas de abortos, según trimestre de gestación, para alpacas positivas a infección por $T$. gondii fue estadísticamente similar a las tasas de abortos de animales que no seroconvirtieron (Cuadro 2), al emplear la Prueba Exacta de Fisher. En forma similar, la mortalidad perinatal encontrada en el estudio fue de $7.5 \%$ (5/67), sin que se observe diferencia estadística entre animales positivos y negativos a infección por T. gondii (Cuadro 3). Asimismo, la infección de $T$. gondii, ajustado por edad, tipo y periodo de gestación no influyeron en el aborto de alpacas gestantes, pero el modelo de ecuación de estimación generalizada (G.E.E) estima que la probabilidad de tener un aborto en el grupo de seropositivas es de 3.3 veces mayor que el grupo de seronegativas a toxoplasmosis. 
Cuadro 1. Infección por Toxoplasma gondii y estado reproductivo (\%) en alpacas del Centro de Investigación y Desarrollo de Camélidos Sudamericanos (CIDCS) Lachocc, Universidad Nacional de Huancavelica. Empadre 1999-2000

\begin{tabular}{cccc}
\hline \multirow{2}{*}{ Infección por $T$. gondii } & \multicolumn{2}{c}{ Estado reproductivo } & \multirow{2}{*}{ Total } \\
\cline { 2 - 3 } & Gestantes & No gestantes & \\
\hline Positivo & $27.6(21 / 76)$ & $18.7(6 / 32)$ & $25(27 / 108)$ \\
Negativo & $72.4(55 / 76)$ & $81.2(26 / 32)$ & $75(81 / 108)$ \\
\hline Total & $70.4(76 / 108)$ & $29.6(32 / 108)$ & \\
\hline
\end{tabular}

$X^{2}=0.330 ; p>0.05$

Cuadro 2. Infección por Toxoplasma gondii y abortos durante los tres tercios de la gestación en alpacas del Centro de Investigación y Desarrollo de Camélidos Sudamericanos (CIDCS) - Lachocc, Universidad Nacional de Huancavelica. Empadre 19992000

\begin{tabular}{lccc}
\hline \multirow{2}{*}{ Infección por T. gondii } & \multicolumn{3}{c}{ Porcentaje de abortos } \\
\cline { 2 - 4 } & $90-180$ días & $181-270$ días & $271-330$ días \\
\hline Positivo & $9.5(2 / 21)$ & $15.8(3 / 19)$ & $12.5(2 / 16)$ \\
Negativo & $5.4(3 / 55)$ & $1.9(1 / 52)$ & $5.9(3 / 51)$ \\
\hline Total & $6.6(5 / 76)$ & $5.6(4 / 71)$ & $7.5(5 / 67)$ \\
\hline
\end{tabular}

Fisher $=0.560 ; p>0.05$

Cuadro 3. Infección por Toxoplasma gondii y mortalidad perinatal en alpacas del Centro de Investigación y Desarrollo de Camélidos Sudamericanos (CIDCS) - Lachocc, Universidad Nacional de Huancavelica. Empadre 1999-2000

\begin{tabular}{cccc}
\hline \multirow{2}{*}{ Infección por $T$. gondii } & \multicolumn{2}{c}{ Porcentaje de mortalidad perinatal } & \multirow{2}{*}{ Total } \\
\cline { 2 - 3 } & Muerto & Vivo & \\
\hline Positivo & $21.4(3 / 14)$ & $78.6(11 / 14)$ & $22.6(14 / 62)$ \\
Negativo & $4.2(2 / 48)$ & $95.8(46 / 48)$ & $77.4(48 / 62)$ \\
\hline Total & $8.1(5 / 62)$ & $91.9(57 / 62)$ & \\
\hline
\end{tabular}

Fisher $=0.071 ; p>0.05$ 
Discusión

El monitoreo de infección por T. gondii se hizo en todas las hembras ( $n=332)$ que ingresaron a la campaña de empadre 1999-2000 en la estación experimental, y posteriormente se siguió con los animales que resultaron negativos a $T$. gondii al inicio del empadre para hacer el seguimiento de la primoinfección, fertilidad (gestaciones) y problemas reproductivos (aborto y mortalidad perinatal).

La posible respuesta inmunitaria disminuida debido al estrés ocasionado por la parición y empadre casi simultáneo de la alpaca (Novoa, 1991; Sumar, 2002), ocasionaría un probable incremento en la receptividad a la infección por $T$. gondii, sumándose a ello los periodos amplios de lactación y gestación de la alpaca. Además, el ecosistema de la puna, donde se encuentra ubicado el CIDCS-Lachocc presenta condiciones ecológicas favorables (SENAMI, 2005), incluyendo la humedad apropiada para la supervivencia de los ooquistes. No obstante, la primera infección ocurre a cualquier edad; muy similar a lo que ocurre en ovinos donde las ovejas jóvenes y adultas son igualmente receptivas a $T$. gondii, siempre que se trate de una primo infección (Luzon et al., 1997).

La seroprevalencia de $T$. gondii de $36.5 \%$ encontrada en el estudio es más baja que la encontrada en la zona sur del país, donde Leguía et al. (1988) y Suárez et al. (2004) reportaron valores de 50 y $45 \%$, respectivamente; mientras que en el centro del país, Poma (2003) encontró valores inferiores (18.8\%). Sin embargo, esta controversia puede ser debida a diferentes factores, incluyendo las diferencias ambientales y climáticas, densidad de población, y técnicas de diagnóstico, pues HAI e inmunofluorescencia indirecta (IFI) varían en sensibilidad y especificidad. Además, la presencia de hospederos definitivos como felinos domésticos y silvestres en las áreas de estudio puede influir en los resultados (Leguía et al., 1988; Poma, 2003).
La evaluación de la dinámica del desarrollo del proceso de infección de $T$. gondii durante la gestación, dividida en tres periodos con intervalos de 90 días, demostró una incidencia acumulada del 25\% (27/108) para los 270 días de observación. No se dispone de trabajos similares en alpacas, pero en borregas uruguayas se reporta una incidencia de $9.8 \%$ (Freyre et al., 1997) y en Nueva Zelandia se encontró incidencias entre 5 a 20\%, dependiendo de los años (1975-1980) (Luzon et al., 1997), resultados que fueron inferiores al presente estudio, debido probablemente a las razones anteriormente señaladas.

Los datos de incidencia por trimestre sugieren la ocurrencia de una hiperendemia a partir del primer periodo del estudio, es decir, una ligera elevación de la frecuencia de la enfermedad (Wayne et al., 1997). Esto fue probablemente ocasionado por el estrés adicional producido por los traslados continuos de las alpacas a zonas bajas que ofrecían instalaciones adecuadas para la toma de muestras de sangre y donde la presencia de gatos era mayor, dada la cercanía al centro poblado de Lachocc. La elevada incidencia de seroconversión podría deberse, además, a la presencia de cepas más virulentas de $T$. gondii. Al respecto, estas cepas tienen fuerte acción patógena en animales, mientras que las cepas de escasa virulencia producirían baja parasitemia y menor invasión tisular (Tenter et al., 2000).

La fertilidad de las alpacas se definió en el presente estudio como la capacidad del animal de quedar gestante. El 70.4\% de animales gestantes, sin diferencia significativa debida a la infección por $T$. gondii, obtenido en el presente estudio, fue similar a otros estudios donde se ha reportado 64.1 y $74.8 \%$ para alpacas de color blanco y de color en la SAIS Tupac Amaru, respectivamente (Gamarra et al., 1985) y de $67.3 \%$ en Puno (Melo, 1996). Un factor que debe haber permitido una elevada tasa de gestación es el uso de empadres alternos o rotatorios (Novoa et al., 1973). 
El 29.6\% (32/108) de las alpacas no quedaron preñadas, y muchas de ellas, probablemente, llegaron a perder el embrión dentro de los primeros 30 días de la gestación. Se tiene evidencia que el 33\% de alpacas primerizas y multíparas sufren de pérdida embrionaria dentro de los primeros 90 días de gestación (Melo, 1996). Así mismo, Fernández Baca (1971) y Novoa (1991) comprobaron que del $80 \%$ de alpacas fecundadas, solamente el $50 \%$ de los óvulos fertilizados sobrevivieron más de 30 días. En el presente estudio, el $18.7 \%$ de las alpacas estaban infectadas con $T$. gondii, lo cual pudo haber repercutido en la supervivencia del embrión; sin embargo, el bajo número de animales afectados no permitió asociar la infección con la mortalidad embrionaria de las alpacas.

Por otro lado, es posible que las pérdidas embrionarias se encuentren sujetas a factores ambientales adversos, tales como el frío y la altura del medio, escasez estacional de alimentos tanto en calidad como en cantidad, enfermedades infecciosas y parasitarias, y alto grado de consanguinidad (Sumar, 2002). Los cambios hormonales en alpacas y llamas señalan un aumento de niveles séricos de estradiol 173 $\mathrm{y}$ un ligero descenso de progesterona entre los días 9 y 11 post ovulación, para luego volver a niveles elevados de progesterona y mantenerse elevados durante toda la gestación (Aba et al., 1995). Lamentablemente, en el presente estudio no se hizo análisis de progesterona al inicio de la gestación para poder determinar pérdidas embrionarias luego de los 11 días de gestación.

Los resultados de abortos (Cuadro 4) en alpacas infectadas naturalmente por $T$. gondii y diagnosticadas mediante ecografía entre los 90-80 y 181-270 días de gestación y en la prueba de balotaje a los 271-330 días fueron de $9.5,15.8$ y $12.5 \%$, respectivamente; siendo estos valores mayores a los presentados por alpacas seronegativas, pero sin diferencia estadística a la prueba de Fischer, posiblemente debido al escaso número de animales empleados.
Se desconoce la proporción de abortos por agentes infecciosos en camélidos domésticos (Sumar 2002); sin embargo, se han reportado dos casos de aborto en llamas por toxoplasmosis en EEUU (Cheney y Allen, 1987), en tanto que Jarvinen et al. (1998) manifiestan que no observaron abortos en dos llamas infectadas con ooquistes. Por otro lado, se ha reportado $27 \%$ de abortos en ovejas primerizas infectadas con ooquistes de $T$. gondii (Blewett et al., 1983). Estas diferencias podrían explicarse con los estudios de Tenter et al. (2000), quienes afirman que la evolución de la toxoplasmosis no es uniforme ni constante en las diversas especies de animales, ni en todos los individuos de una misma especie.

En el Perú, las elevadas tasas de mortalidad por causas infecciosas, principalmente en crías, constituye un factor limitante en la crianza de alpacas (Ramírez, 1991). Ameghino y DeMartini (1991) refieren que la toxoplasmosis sería responsable de la alta mortalidad perinatal observada en los camélidos sudamericanos; lo cual podría coincidir con la tasa de mortalidad perinatal en animales seropositivos de $21.4 \%$ encontrada en el presente estudio en comparación con el $4.2 \%$ en animales seronegativos, pese a que no se llegó a observar una diferencia estadística entre estos promedios (Cuadro 3). Por otro lado, las causas de muerte fetal por infecciones intrauterinas se deberían a agentes bacterianos, virales, hongos y protozoarios (Ramírez, 1991; Ameghino y DeMartini, 1991).

Dubey y Kirkbride (1989) mencionan que el feto y las crías débiles recién nacidas pueden morir como consecuencia de graves lesiones originadas por la colonización y multiplicación de un agente infeccioso en la placenta, afectando la trasferencia de oxigeno al feto, lo que ocasiona lesiones cerebrales. Al respecto, Rhyan y Dubey (1984) afirman que algunos casos de hipoxia fetal se verían agravados por acción de sustancias toxicas liberadas en la destrucción de la placenta, además que lesiones placentarias severas 
puede alterar el equilibrio hormonal necesario para el mantenimiento de la gestación, favoreciendo el aborto.

En otros estudios sobre enfermedades parasitarias productoras de infertilidad se ha reportado la presencia de Neospora caninum en $38 \%$ de fetos abortados de alpacas y llamas en el sur y centro del Perú, a través de pruebas de inmunohistoquímica, PCR e histopatología (Serrano, 2005); sin embargo, no encontraron $T$. gondii en dichas muestras.

\section{Conclusiones}

- No se halló asociación de la infección de $T$. gondii con abortos ocurridos en el $1^{\text {er }}, 2^{\text {do }}$ y $3^{\text {er }}$ trimestre de la gestación en alpacas.

- Se observó una mayor frecuencia de abortos en los tres trimestres de la gestación y de mortalidad perinatal en alpacas seropositivas que en seronegativas a $T$. gondii aunque sin diferencia estadística. Sin embargo, la prueba de Odds Ratio indicó que las alpacas seropositivas presentan 3.3 veces mayor riesgo de aborto que las seronegativas.

\section{Literatura Citada}

1. Aba M, Forsberg M, Kindahl H, Sumar J, Edqvist L. 1995. Endocrine changes alter mating in pregnant and nopregnant llamas y alpacas. Acta Vet Scand 36: 489-498.

2. Ameghino E, DeMartini J. 1991. Mortalidad en crías de alpacas. IVITAPCAIRM: Lima. p 1-121.

3. Ameguino E, Rivera S, Zaferson J, Velarde N, Chávez E, Arroyo J, DeMartini J. 1991. El aspecto sanitario en alpacas y ovinos de las comunidades del departamento de Puno. Cusco:
Anales V Convención Internac Camélidos Sudamericanos. p 142.

4. Blewett DA, Bryson CE, Miller JK. 1983. Studies of antibody titres in experimentally induced ovine toxoplasmosis. Res Vet Sci 34: 163-166.

5. Cheney JM, Allen GT. 1987. Parasitism in llamas. Vet Clin North Am: Food Anim Pract 5: 217-225.

6. Daniel WW. 2004. Bioestadística. Base para el análisis de las ciencias de la salud. $4^{\text {ta }}$ ed. México: Ed. LIMUSA. 915 p.

7. Dubey JP, Kirkbride CA. 1989. Economic and public health considerations of congenital toxoplasmosis in lambs. J Am Med Assoc 195: 1715-1716.

8. Fernández Baca S. 1971. La alpaca: reproducción y crianza. Bol. Div. N. ${ }^{\circ} 7$. IVITA, Perú. 43 p.

9. Freyre A, Bonino J, Falcon J, Castells D, Correa O, Casaretto A. 1997. The incidence and economic significance of ovine toxoplasmosis in Uruguay. Vet Parasitol 73(1-2): 13-15.

10. Gamarra M, Galarza E, Quintana F, Cabrera P. 1985. Tasas de fertilidad, natalidad y mortalidad en alpacas. En: Libro de Resúmenes VIII Reunión Científica APPA. Huancayo: APPA. F-18.

11. Jarvinen J, Dubey JP, Althouset CG. 1998. Clinical and serologic evaluation of two llamas (Lama glama) infected with Toxoplasma gondii during gestation. J Parasitol 85: 142-144.

12. Leguía G. 1990. Toxoplasmosis en alpacas. Bol Div N. ${ }^{\circ} 23$. Lima: Univ Nac Mayor de San Marcos.

13. Leguía PG, Samame BH, Guerrero DCA, Rojas CM, Nuñez LA. 1988. Prevalencia de anticuerpos contra Toxoplasma gondii en alpacas. Rev Camélidos Sudamericanos 6: 19-22.

14. Luzon M, Miro G, Quintanilla A. 1997. Epidemiología. En: Toxoplasmosis Neosporosis. Madrid, España: Ed. Lusan. p 19- 32.

15. Melo M. 1996. Influencia de la edad, el estado nutricional, la condición hormonal y el manejo de las alpacas sobre la 
sobre vivencia embrionaria. Allpaka IIPC 5(2): 26-46.

16. Melo M. 1997. Sistemas de control y manejo sanitario de alpacas y llamas en la región andina del sur peruano. Puno, Perú: Universidad Nacional del Altiplano. 123 p.

17. Novoa C. 1991. Fisiología de la reproducción de la hembra. En: FernándezBaca S (ed). Avances y perspectivas del conocimiento de los camélidos sudamericanos. Santiago de Chile: Oficina Regional FAO para América Latina y el Caribe. p 91-107.

18. Novoa C, Sumar J, Leyva V, Fernández Baca S. 1973. Incremento reproductivo en alpacas de explotaciones comerciales mediante metodo de empadre alternado. Rev Inv Pec (IVITA) 2(2): 191-193.

19. Poma E. 2003. Seroprevalencia de anticuerpos contra Toxoplasma gondii en alpacas (Lama pacos) de la Unidad de producción de Cochas de la SAIS Tupac Amaru. Tesis de Médico Veterinario. Lima: Facultad de Medicina Veterinaria, Univ. Nacional Mayor de San Marcos. 58 p.

20. Puma G, Garnica J, Bravo W. 1999. El tamaño de la placenta, la edad de la madre y la supervivencia perinatal de la cría alpaca. En: Resumen de II Congreso Mundial sobre Camélidos. Cusco. p 103.

21. Ramírez A. 1991. Enfermedades infecciosas. En: Fernández Baca S (ed). Avances y perspectivas del conocimiento de los camélidos americanos. Santiago de Chile: Oficina Regional FAO para América Latina y el Caribe. p 334-337.

22. Rhyan JC, Dubey JP. 1984. Ovine abortion and neonatal death due to toxo- plasmosis in Montana. J Am Vet Med Assoc 184(6): 661-664.

23. Rosadio R, Ameguino, E. 1999. Enfermedades de los ovinos en el Perú. Púb Téc FMV No 40. Lima: Facultad de Medicina Veterinaria, Univ. Nacional Mayor de San Marcos. 76 p.

24. SENAHMI. 2005. Estación meteorológica Callqui-Huancavelica. Registro de temperatura y precipitación pluvial. Lima: SENAHMI.

25. Serrano M. 2005. Identificación de Neospora caninum en abortos de camélidos sudamericanos domésticos del Perú. Tesis de Magíster. Lima: Facultad de Medicina Veterinaria, Univ. Nacional Mayor de San Marcos. $111 \mathrm{p}$.

26. Suárez F, Flores W, Chávez A, Rivera H, Huanca W. 2004. Toxoplasmosis en la sierra alto andina. Rev Inv Vet, Perú 15: 170-173.

27. Sumar J. 2002. Llamas y alpacas. En: Hafez E, Hafez B (eds). Reproducción e inseminación artificial en animales. México: Ed. McGraw-Hill. p 224-242.

28. Tenter A, Heckeroth A, Weiss L. 2000. Toxoplasma gondii: from animals to humans. Int J Parasitol 30: 1217-1258.

29. Velásquez VC, Novoa MC. 1999. Superovulación con PMSG aplicada en fase folicular y fase luteal en alpacas. Rev Inv Vet, Perú 10(1): 39-47.

30. Wayne S, Meek A, Willeberg P. 1997. Epidemiología Veterinaria: principios y métodos. Zaragoza, España: Ed. Acribia. $384 \mathrm{p}$.

31.Zeger SL, Liang KY. 1986. Longitudinal data analysis for discrete and continuous outcomes. Biometrics 42: 121-130. 